\title{
THE LICK OBSERVATORY HAMILTON ECHELLE SPECTROMETER
}

\author{
Steven S. Vogt \\ Lick Observatory and Board of Studies in Astronomy \\ and Astrophysics \\ University of California, Santa Cruz, CA 95064 .
}

\begin{abstract}
The Hamilton Echelle Spectrometer, recently installed at the coudé focus of the Shane 3-m telescope, is a high dispersion spectrograph optimized for use with large format CCD's. It was designed primarily for high resolution $(R=50,000)$ wide bandpass spectroscopy of point-like sources down to a limiting magnitude of about $\mathrm{V}=16.5$, over the $0.34 \mu \mathrm{m}$ to $1.1 \mu \mathrm{m}$ spectral region. Its design features a relatively large collimated beam size, the use of prisms rather than gratings for cross dispersion, minimum order separation, the use of protected silver mirror coatings throughout the system, and a fast (f/1.67) folded Schmidt camera with a flat external focal plane. Together, these features yield a very powerful spectrometer for high resolution stellar spectroscopy. This paper gives a brief description of the Hamilton spectrometer and gives several examples of its performance on astronomical objects.
\end{abstract}

\section{DISCUSSION}

The Hamilton Echelle is a prism-cross-dispersed echelle spectrometer recently commissioned at coudé of the 3-meter telescope. It was optimized for today's largest format CCD detectors and for the even larger format CCD's anticipated in the next few years. This paper presents a very brief description of the spectrometer. A more detailed discussion of the instrument and the philosophy behind its design will appear elsewhere.

Figure 1 shows the layout of the Hamilton in top and side views. The $\mathrm{f} / 36$ beam from the $3-\mathrm{m}$ telescope enters the slit (1) at upper right. The beam is collimated into a $20.4 \mathrm{~cm}$ beam by an off-axis parabolic collimator (2) and deviated $11^{\circ}$ to an echelle grating (3). The echelle is a $204 \times 408 \mathrm{~mm} \mathrm{R}-2$ with 31.6 $\mathrm{gr} / \mathrm{mm}$, purchased from Milton Roy Inc. (formerly Bausch and Lomb). It is used in-plane $\left(\gamma=0^{\circ}\right)$ with $\theta=5.5^{\circ}$. The light is then cross dispersed by two large UBK-7 prisms (4) and (5). Prisms were chosen over gratings since they provide 
higher throughput, lack of blaze fall-off, and much more uniform order separation. The latter is particularly important since real estate on the CCD detector is precious. Order separation with gratings varies substantially with wavelength, resulting in much wasted detector area between orders. Prisms yield approximately constant order separation over the entire visual and near IR spectrum. The Hamilton was designed for minimum order separation, thus maximizing spectral coverage per observation.

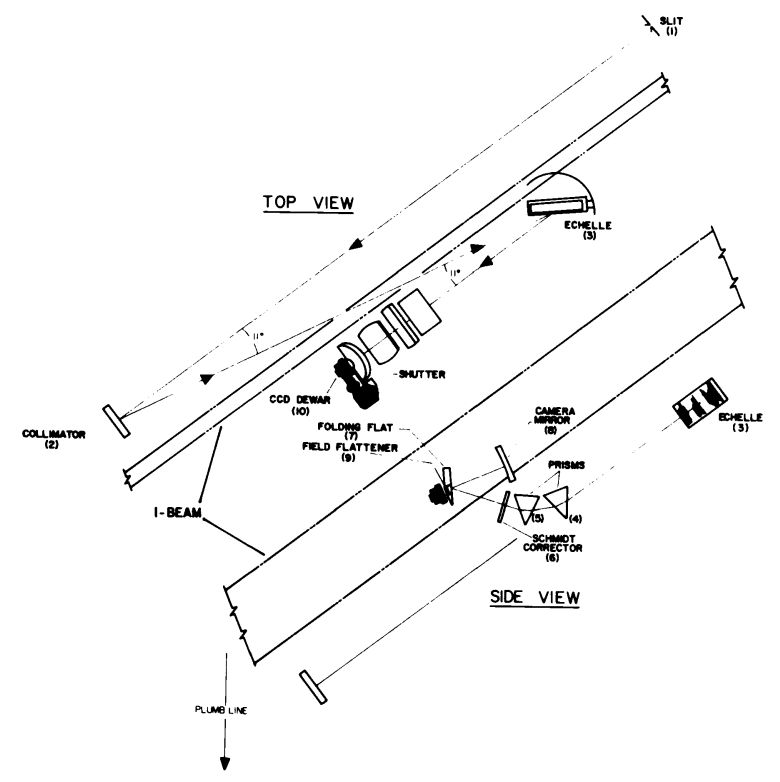

Figure 1. Hamilton spectrometer in top and side views.

After cross dispersion, the light is brought to focus by an $f / 1.67$ (polychromatic) folded Schmidt camera designed by Dr. Harlan Epps at the University of California at Los Angeles. This camera features a flat, external focal plane located $2.3 \mathrm{~cm}$ beyond the last optical surface. As such, it reaches easily into a typical CCD dewar. The focal surface is flat, and no refocussing is required over the entire $3400 \AA$ to $11,000 \AA$ range. The camera consists of a Schmidt corrector (6), a folding flat (7), a spherical camera mirror (8), and a field flattener (9) mounted in the hole in the folding flat. At present, the principle detector for the Hamilton is a thinned backside illuminated Texas Instruments $800 \times 800 \mathrm{CCD}$ with readout noise of about 7 electrons. A large mounting stage behind the camera is provided to allow other detectors (MAMA's, PAPA's, Ranicons, etc.) to be used with the Hamilton.

The Hamilton's relatively large beam size and high blaze angle echelle $\left(\theta_{b}=64.7^{\circ}\right)$ allow a relatively large slit to be used, even at high resolutions. A 
1.25 arc-sec slit projects down to two 15 micron CCD pixels. The 'two-pixel' resolution equivalent is 60,000 and the true effective resolution with a 'two-pixel projected slit' is about 40,000 or $0.125 \AA$ at $5000 \AA$.

All the reflective optical surfaces of the Hamilton have been optimized for highest efficiency in the visual/near-IR region by using protected silver coatings. The silver coatings were done in the Lick Coating Laboratory. The silver is laid down $1000 \AA$ thick on a protective underlayer of $400 \AA$ of copper. A 1000 $\AA$ thick overcoat of sapphire is then applied by e-beam deposition to seal the silver from exposure to the atmosphere, thus preventing tarnishing and protecting against mechanical abrasion. All transmitting optical surfaces are coated with single layer $\mathrm{MgF}$ anti-reflection coatings.

Figure 2 shows the optical format of the Hamilton. Each horizontal line in the format represents a single order, and the length of each line represents one free spectral range. A few order numbers are shown at the left of each order, and blaze wavelengths are at the right. Our present TI CCD (format shown by the inner box) does not cover the entire format, but it nevertheless spans typically $800 \AA$ to $3600 \AA$ in a single exposure. The addition of a Tektronix $1024 \times 1024$ (format shown by the outer border) will bring the Hamilton to full maturity, providing complete spectral coverage from $3400 \AA$ to $9100 \AA$ in a single exposure with no interorder gaps, a glorious match between echelles and CCD's.

HAMILTON ECHELLE FORMAT

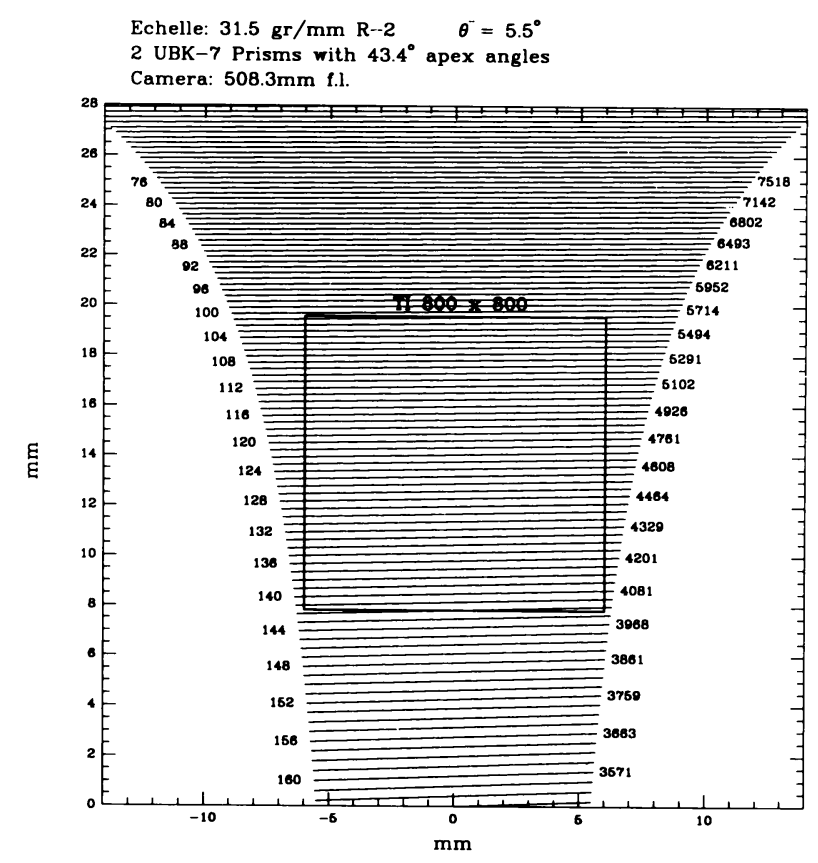

Figure 2. Hamilton spectral format. 
Figure 3 shows a Hamilton spectrum of the $V=12.3$ magnitude globular cluster star M71-A4, obtained with the $800 \times 800$ CCD. This star was selected as a good benchmark test of the system performance since it had already been studied with echelles or other spectrographs on several different large telescopes in recent years. The horizontal white bars are the spectral orders of the echelle. The spectrum runs from $4530 \AA$ at the bottom to $7320 \AA$ at the top. The atmospheric B-band is visible near row $80 . \mathrm{H} \alpha$ is the prominent absorption feature near row 135. The strong pair of lines at row 285 are the sodium $D$ lines. The strong line to the left at row 495 is part of the $\mathrm{Mg} \mathrm{b}$ feature. This 30 minute exposure yielded a resolution of $R=30,000(0.22 \AA)$ and a $S / N$ of 150 per resolution element at $\mathrm{H} \alpha$.

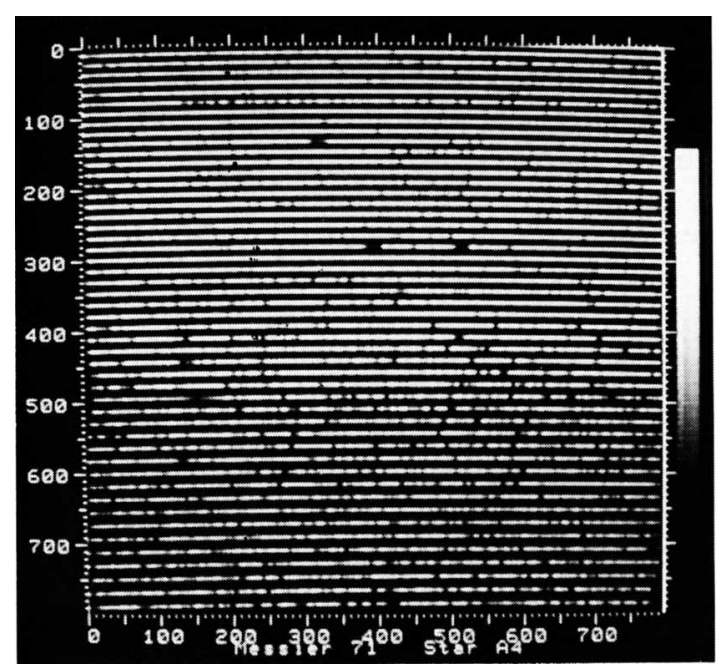

Figure 3. Hamilton spectrum of M71-A4.

Figure 4 shows a performance comparison between the Hamilton and telescope/spectrometer combinations at Kitt Peak and Palomar. The object is again the globular cluster star M71-A4 in the region around $6200 \AA$. The top spectrum (Cohen 1983, Ap.J., 270, 654) shows a 2.3 hour exposure obtained with the Kitt Peak 4-m telescope Cassegrain Echelle Spectrograph with the Singer image tube camera and baked IIIa-J plates. A resolving power of about 12,500 was achieved here. The middle spectrum (from the same reference) shows a 1 hour exposure with the Palomar 5-m telescope using the red side of the Double Spectrograph, and a TI $800 \times 800$ CCD. A resolution of 9,500 was achieved here. The lower spectrum shows the corresponding spectral region extracted from the 30 minute Hamilton exposure of Figure 3. The Hamilton data have been smoothed to a resolution of about 20,000 here. The Hamilton thus obtained 2 to 3 times the resolution in one-half to one-quarter the observing time, using a telescope 
with only one-half to one-third the collecting area. This comparison illustrates the great strides in performance that are now achievable in high resolution spectroscopy because of technological advances in detectors, coatings, and optical glasses.

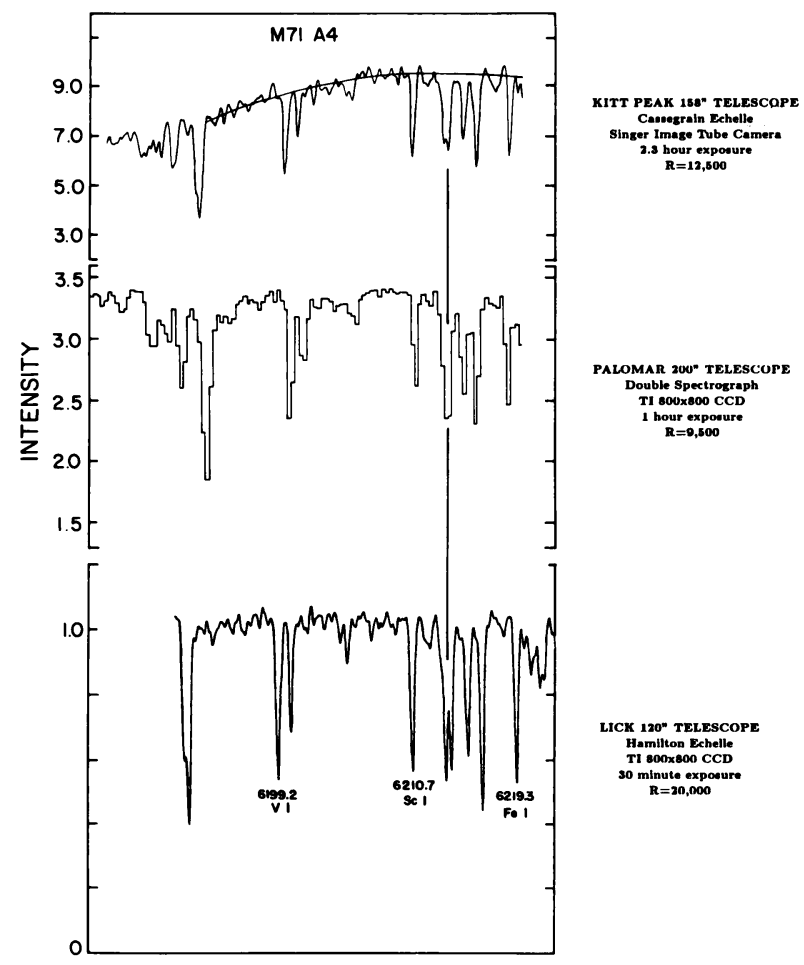

Figure 4. Performance comparison

As an example of the Hamilton performance near its present limiting magnitude, Figure 5 shows a 240 minute observation of the $B=16.0$ magnitude QSO $1331+170$. This is the sum of two 2-hour exposures taken under very good conditions on $4 / 17 / 87$ UT. The CCD was binned $2 \times 2$ on-chip to reduce the readout noise contribution. Shown here is only a subsection of the original $400 \times 400$ pixel binned image (the areas not shown contain hot pixels and hot columns which became heavily saturated in the long exposures). Most of the cosmic ray hits have been removed by median filtering. The 2.25 arcsecond entrance slit projected to 1.8 binned pixels yielding an effective resolution of about 2.5 pixels $(0.2 \AA)$ or resolving power of 24,000 . The section shown covers the wavelength range from about $3985 \AA$ at the bottom to $5020 \AA$ at the top. At the blue end the wavelength coverage is about $75 \%$ complete, dropping to $60 \%$ complete at the red end. The effective $\mathrm{S} / \mathrm{N}$ is about 8-12 per pixel or 13-19 per resolution element. The strong night sky line just below center at row 202 is $\mathrm{Hg} \mathrm{I} \lambda 4358 \AA$, 
and the two weaker night sky lines near the bottom of the chip are $\mathrm{Hg}$ I $\lambda 4078 \AA$ (row 299), and $\mathrm{Hg}$ I $\lambda 4047 \AA$ (row 310). Numerous absorption features are visible in the QSO spectrum. The very strong complex of absorption features to the right of center near row 221 is the C IV $\lambda \lambda 1548,1551 \AA$ doublet at $\mathrm{z}=1.7755$. Two other strong absorption features from the same system are visible; Si II $\lambda$ $1527 \AA$ just below the C IV complex at row 241 , and Al II $\lambda 1671 \AA$ left of center near row 124. The complex of absorption features near row 69 is the $\mathrm{Mg}$ II $\lambda \lambda$ $2795,2803 \AA$ doublet at a redshift of $\mathrm{z}=0.7441$.

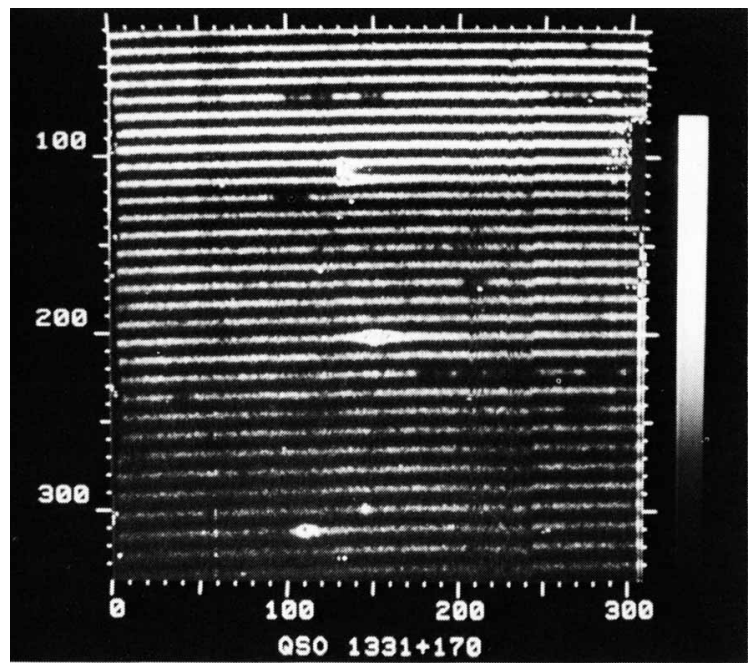

Figure 5. Hamilton spectrum of QSO $1331+170$.

Figure 6 shows the reduced spectrum from echelle order 117 near row 69 of Figure 5 which contains the Mg II doublet. At the present resolution of 0.20 $\AA$, the $\mathrm{Mg}$ features are resolved into at least 8 separate components which span a velocity range of some $260 \mathrm{~km} \mathrm{~s}^{-1}$. These different components probably arise from discrete absorbing clouds moving with different velocities in a distant intervening galaxy along the line-of-sight to the QSO. Data such as these will make detailed metal abundance studies possible in very distant galaxies over a large range of look-back times. Here, high resolution is crucial for distinguishing abundance effects from multiple velocity shifted cloud components, both of which contribute to the total equivalent width.

As performance benchmarks for the Hamilton and our TI $800 \times 800$ $\mathrm{CCD}$, one obtains $\mathrm{S} / \mathrm{N}=45$ per $0.15 \AA$ resolution element at blaze center near $5500 \AA$ in a 1 hour exposure at $\mathrm{V}=13.0$ under excellent conditions and gray time. At the faint end, under similar conditions, the Hamilton delivers $\mathrm{S} / \mathrm{N}=10$ per 0.2 $\AA$ resolution element in 4 hours at $\mathrm{V}=17.0$. At this level, dark current and CCD 
readout noise become major contributors to the noise.

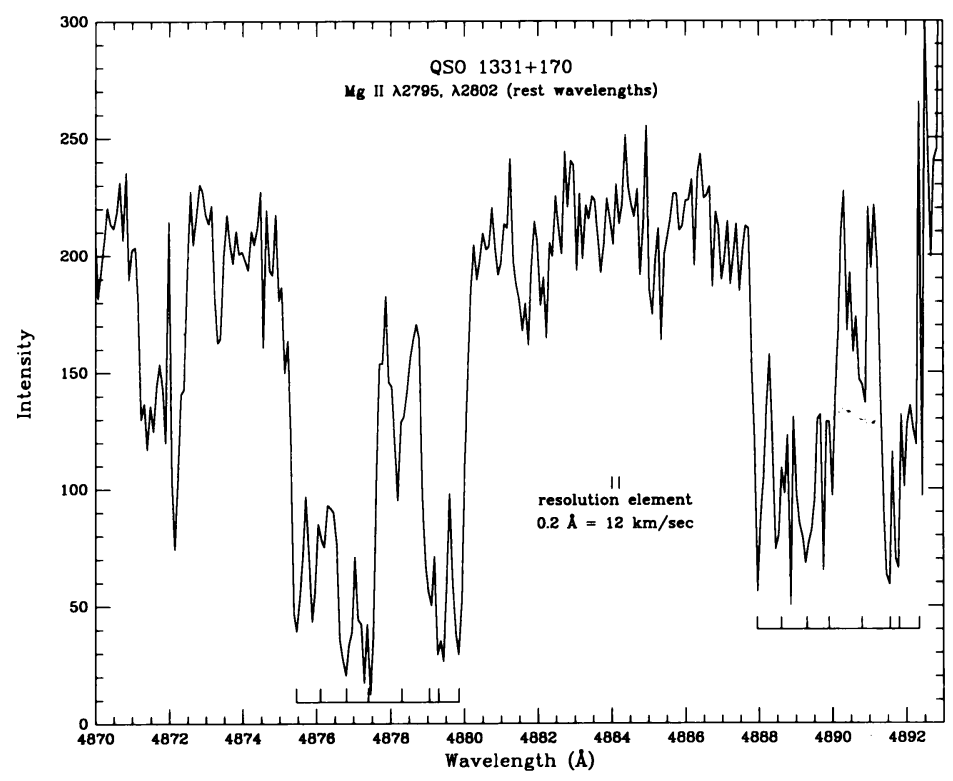

Figure 6. Reduced spectrum of $\mathrm{Mg}$ II doublet.

\section{DISCUSSION}

SMITH: Do you need different exposure times for different wavelength regions because of the large flux gradients?

VOGT: Yes, of course. When working on cool stars, there is an enormous flux gradient between the UV/blue and the near-IR regions. The dynamic range of the CCD is quite large, but to optimize exposures at such wavelength extremes, two exposures are advised.

EBBETS: Does the close order spacing create difficulties with measuring and removing background and scattered light?

VOGT: Not really. To first order, the orders are just far enough apart where background correction and scattered light removal are straightforward. However, we have not yet completed our software routines for data reduction. I am sure that, as we push to high accuracy, and study the effect more carefully, we will find it necessary to implement some more sophisticated approaches to the task. 